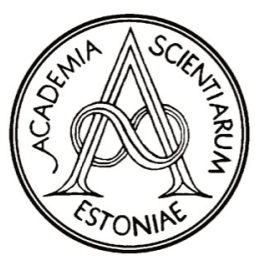

Proceedings of the Estonian Academy of Sciences, $2021,70,3,286-296$

https://doi.org/10.3176/proc.2021.3.07

Available online at www.eap.ee/proceedings

MULTI-BODY

DYNAMICS

\title{
Tri-planar balancing optimization of a double crank-rocker mechanism for shaking forces and shaking moments reduction
}

\author{
Anwr M. Albaghdadi*, Masri B. Baharom and Shaharin A. Sulaiman \\ Department of Mechanical Engineering, Universiti Teknologi PETRONAS, 32610 Seri Iskandar, Perak Darul Ridzuan, Malaysia
}

Received 26 April 2021, accepted 25 June 2021, available online 12 August 2021

(C) 2021 Authors. This is an Open Access article distributed under the terms and conditions of the Creative Commons AttributionNonCommercial 4.0 International License (http://creativecommons.org/licenses/by-nc/4.0/).

\begin{abstract}
In this study, a new optimization approach is introduced to reduce vibration exerted on a double crank-rocker mechanism. A dynamic analysis based on planar methods of this mechanism is suggested, and formulation of shaking forces and shaking moments inducing vibration on this system is presented. A two-step optimization technique is suggested to study system sensitivity to the components of shaking forces and moments to enhance the mechanism's balancing process. This leads to identification of the most dominant parameters which are then used to formulate the objective functions of effective optimization. Each objective function is studied for each individual case, after which the outcome results of the mechanism's balancing optimization are introduced and discussed.
\end{abstract}

Key words: crank-rocker mechanism, balancing optimization, shaking forces, shaking moments.

\section{INTRODUCTION}

As regards the problem of the trade-off between shaking forces and shaking moments balancing of a four-bar mechanism, many studies have been conducted to minimize this gap and search for an optimal solution that suits each distinct case. Some of these studies are related to full or partial optimization to reduce both shaking forces and moments, while others are only concerned with one part of the problem at a time. In mechanical systems where forces are balanced using methods such as mass distribution or counterweight addition, the increment of shaking moment values is definite since it is affected by mass inertia [1]. Consequently, scholars have studied methods for shaking forces and moments reduction by adding counterweights to minimize their undesired effect on related systems.

Berkof [2] suggested a combination of redistribution of linkage masses and addition of counterweights to fully balance a four-bar linkage system even under variable speeds. In the proposal, the external loads were not considered during optimization. The cost of the proposal was the increase in the system's total mass and input torque. Also, Lowen and Berkof [3] studied shaking moment optimization of a force-balanced four-bar mechanism. It was concluded that there were many factors that affected the optimal optimization of a specific system such as linkage length ratios, mass and density characteristics, and the input angular velocity.

Some limitations were also present when performing the optimization process, which needed to be taken into consideration. Of these limitations, there were more than one optimization minima, of which only one could be

\footnotetext{
*Corresponding author, al.bghdady@gmail.com
} 
attained. Moreover, applying the concept of optimal mass distribution of a mechanism is only limited to specific configurations. The ability to eliminate forces through different means can lead to an inevitable increase in moment values, owing to many reasons such as the increase in the system's total mass. Arakelian [4] presented a method for shaking moment balancing of self-balanced double-acting slider-crank mechanisms. It was noticed that the reaction forces were cancelled out due to a specific mechanism arrangement. However, the shaking moments were still present, which could lead to undesired vibration. The author suggested that shaking moments could be eliminated using the combination of the effect of pantograph linkage and application of a mass redistribution of the coupler link. As a result, both the shaking moment and input torque were optimized.

To eliminate the vibration induced by shaking moments from another perspective, Acevedo et. al. [5] suggested a redundant motor to counter the inertial mass of a previously force-balanced four-bar linkage. This method is easier in terms of application, and it prevents any mass increment of the system or complexity in the original configuration. Moreover, this method can be applied to a wider range of working mechanisms under the condition of the system's pre-balanced reaction forces.

The balancing of a spatial mechanism has also been considered and studied on different occasions. Arakelian [6] presented a simplified method to achieve complete balancing of spatial linkages related to forces and moments caused by inertial loads. The author suggested replacing the original configuration of the coupler by two concentrated masses on both ends. This would transform the balancing method into rotating linkages with concentrated masses. Several researchers have performed dynamic analyses and balancing of spatial multibody systems by means of comprehensive computational study [7] while others [8,9] have proposed different balancing methods through the development of optimization techniques to enhance such systems.

In this paper, the problem of the trade-off between shaking forces and shaking moments exerted on a double crank-rocker mechanism is introduced. This problem was studied earlier in [10], where the two-dimensional dynamic analysis was only introduced. Hence, the objective of this research was to perform a study to minimize the vibration acting on a double crank-rocker (DCR) mechanism considering a three-dimensional dynamic analysis. The proposed method involves a trade-off between shaking force and shaking moment balancing. This elementary mechanism architecture is suggested to overcome vibration resulting from shaking forces and moments, mainly due to its ability to counter the inertial action between different mechanism linkages, similar ideas were reported in [1]. The method of using counterweights for shaking force balancing was applied and the optimization of this system is presented. However, due to this process, an increase in the system's mass led to the increase in shaking moments. Even though this unique configuration of a DCR mechanism was expected to reduce the overall shaking moments, further enhancement could be introduced to achieve optimal results. A simplified approach of the triplanar analysis of different forces and moments is introduced, which is then used to perform a three-dimensional balancing optimization process. In addition, a developed method applying the best objective function for optimal balancing optimization is suggested, followed by a comparison between optimization results using enhanced objective functions.

\section{DOUBLE CRANK-ROCKER TRI-PLANAR DYNAMIC ANALYSIS}

In this section, a dynamic analysis of a DCR mechanism is introduced, as well as a 3-dimensional configuration and a planar based study of this system is presented. Figure 1 shows the basic configuration of the DCR mechanism along with kinematic representation. The geometry of linkages is represented by numbers (1 to 6), the corresponding angles between the links $(\theta, \Upsilon, \varphi)$ are a crank angle, transmission angle and throw angle, respectively. These parameters were identified using methods presented in the previous study [11]. In addition, counterweights used for force balancing are represented by letters (a, b, c, and d), and joint reaction forces are shown on two planes (y-x and $\mathrm{z}-\mathrm{x}$ ).

Many methods for determining reaction forces acting on a planar four-bar mechanism have been discussed in previous studies. However, in this study we adopted the well-known methods mentioned in [12-15]. The essence of these studies was to apply the theory of the system's virtual work to find all the forces acting on different linkages which are the result of summing up of external forces, linkages' internal and transitional momentum forces. The 
(a)

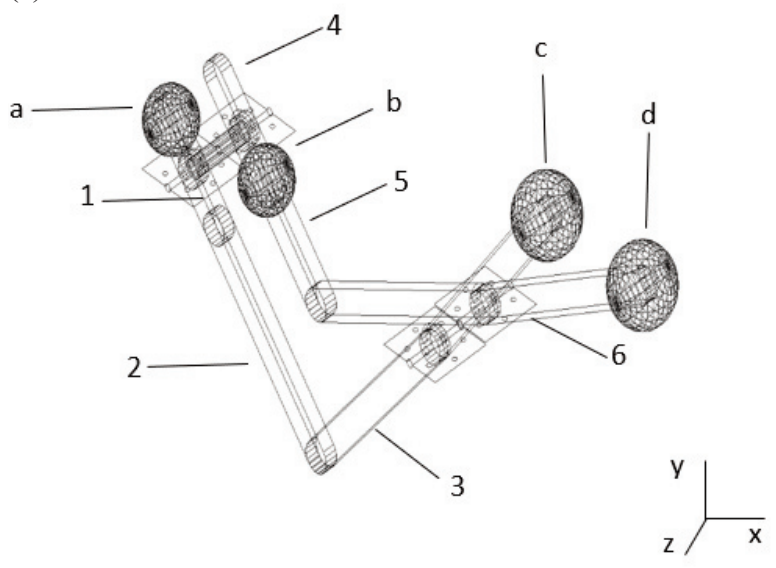

(b)

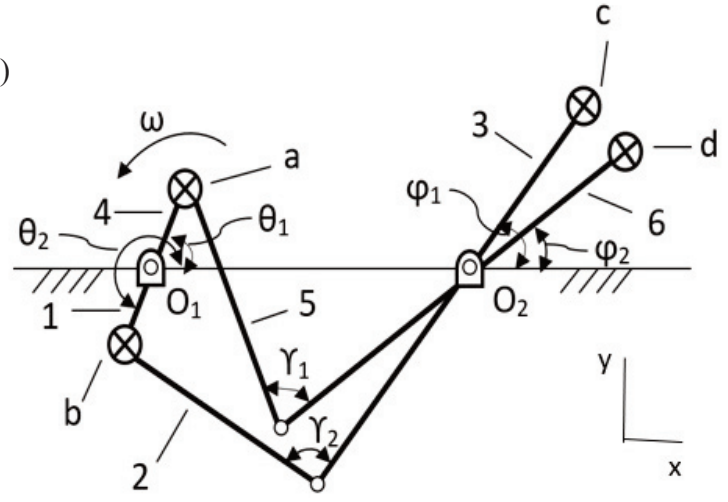

(c)

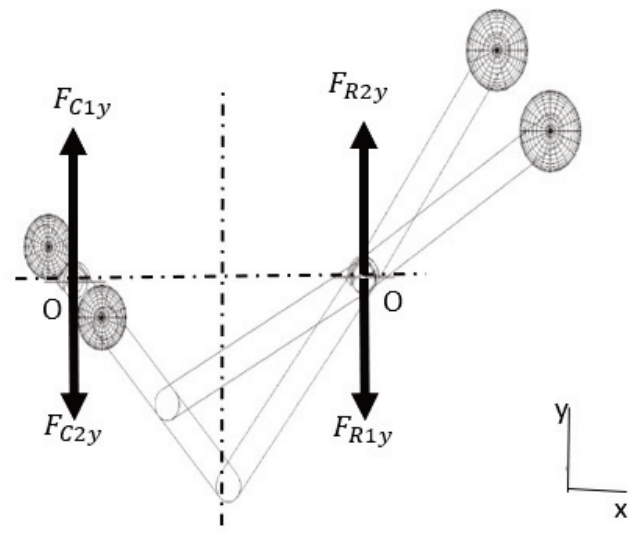

Fig. 1. Configuration and dynamic analysis of DCR mechanism: 3D configuration (a), kinematic scheme (b), plan view y-z (c), and plan view z-x (d).

resultant forces were then translated into equivalent reaction forces acting on the ground pivot. By applying this method to the DCR mechanism, the joint shaking forces desired for a balanced system could be expressed by:

$$
\begin{aligned}
& F_{x}=F_{C 2 x}+F_{R 1 x}-\left(F_{C 1 x}+F_{R 2 x}\right), \\
& F_{y}=F_{C 1 y}+F_{R 1 y}-\left(F_{R 2 y}+F_{C 2 y}\right),
\end{aligned}
$$

where $F_{x}$ and $F_{\mathrm{y}}$ are shaking forces on $\mathrm{x}$ - and y-directions and defined positive according to the coordinate directions. Furthermore, $F_{C 1}$ and $F_{C 2}$ represent reaction forces by the crank linkages 1 and 4 respectively, on the ground pivot $\mathrm{O}_{1}$. Similarly, $F_{R 1}$ and $F_{R 2}$ are reaction forces by the rocker linkages 3 and 6 respectively, on the ground pivot $\mathrm{O}_{2}$. However, only forces in the $\mathrm{x}$ - and $\mathrm{y}$-directions were considered since forces acting in the z-direction have a negligible value, as indicated in Fig. 2.

To determine reaction moments, a simplified approach was introduced by extending the planar analysis method into a multi-planar analysis and then the resultant moment in each plane was concluded. Also, only moments caused by shaking forces were considered, where moments caused by inertia were included in calculations of the reaction forces of joints. Moreover, mechanism inertial moments were expected to be highly balanced by means of mechanism duplication. In this study, three moment components which are acting in three planes (y-x, z-x and y-z) were considered, namely $M_{z}, M_{y}$ and $M_{x}$ respectively, and they could be found by calculating moments at point $\mathrm{G}$ that mediate ground pivots on the z-x plane, Fig. 1. Assuming a clockwise moment as the positive direction in balancing this mechanism, the calculations to find the total moment $M_{Z}$ resulting from forces acting on the z-x plane are presented by:

$$
M_{z}=\frac{O_{1} O_{2}}{2} \times\left(F_{C 1 y}-F_{C 2 y}+F_{R 1 y}-F_{R 2 y}\right),
$$



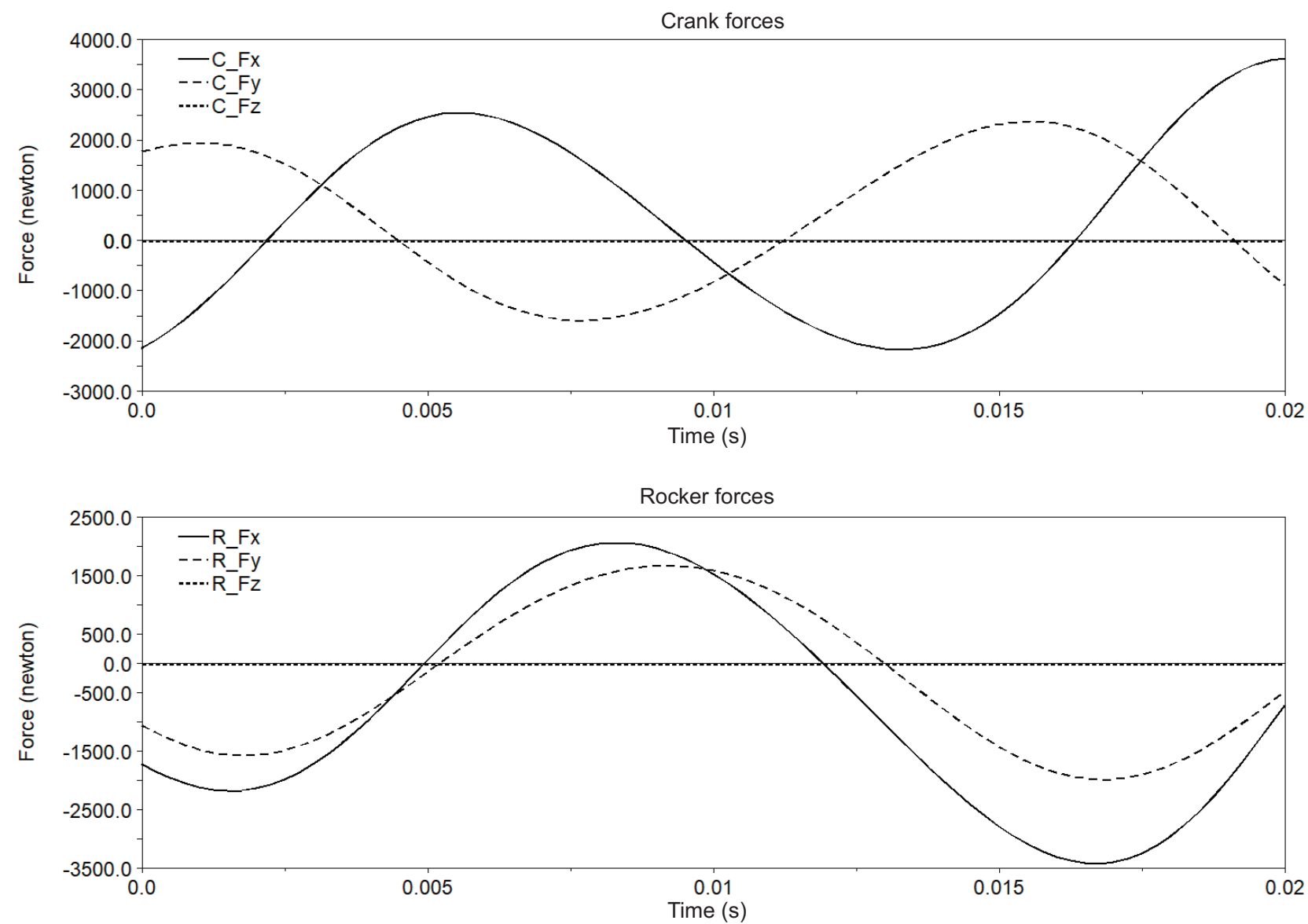

Fig. 2. Crank and rocker shaking forces.

where $O_{1} O_{2}$ is the distance between ground pivots of the DCR mechanism. Similarly, the moment $M_{y}$ at point G can be found by:

$$
M_{y}=\frac{A_{1} A_{2}}{2} \times\left(F_{C 1 x}+F_{C 2 x}-F_{R 1 x}-F_{R 2 x}\right),
$$

where $A_{1} A_{2}$ is the space distance between both the 1 st and the 2 nd DCR mechanism components. And for the plane $\mathrm{y}-\mathrm{z}$, the moment $M_{x}$ caused by shaking forces in the y-direction can be expressed by:

$$
M_{x}=\frac{A_{1} A_{2}}{2} \times\left(F_{R 1 y}+F_{R 2 y}-F_{C 1 y}-F_{C 2 y}\right) .
$$

As suggested earlier, the effect of the shaking force $F_{z}$ was not considered due to negligible values. Moments acting on an unbalanced system are shown in Fig. 3, indicating the dominance of the shaking moment $M_{z}$ compared to $M_{x}$ and $M_{y}$.

\section{OPTIMIZATION PROCESS}

Previously, an optimization study based on a planar dynamic analysis was discussed and validated for balancing a DCR mechanism [10]. In this study, the aforesaid method was adopted and introduced to investigate the effectiveness of implementing the three-dimensional dynamic approach. Usually, the optimization process starts with identifying the system components, system constraints, design variable and objective function [16]. As there 


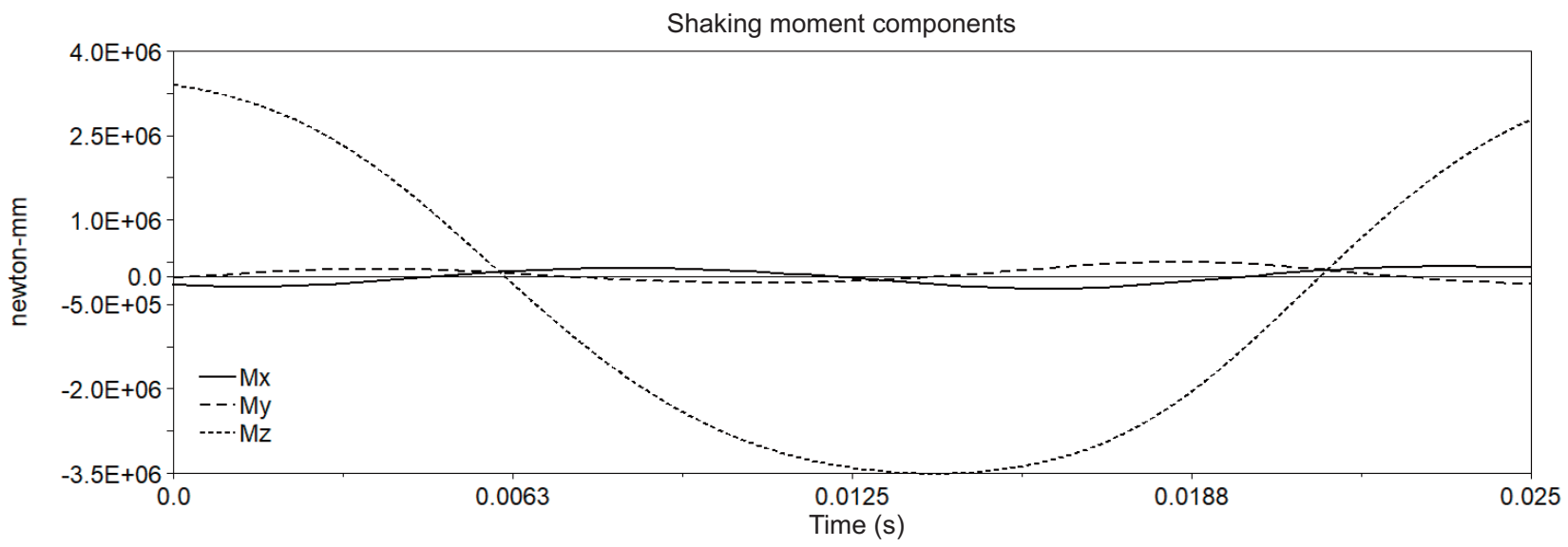

Fig. 3. Total shaking moment.

are many different approaches to identify the desired objective functions, in this study a practical approach was suggested to present an effective objective function based on studying the impact of the sub-components of dynamic shaking forces and shaking moments. For the DCR mechanism, it was desired to achieve better results when performing optimization on such systems. Thus, two-step identification of different objective functions was implemented - the first step was based on analysing the shaking forces and moments acting on this mechanism, and then the second step was to compare the results obtained from the first step. The functions which contributed to the best results were selected for the optimization process. The elements considered for the optimization objective functions (OFs) were forces presented in the equations (1)-(4). Introducing the root mean square (RMS) of the total forces led to the following expression [17,18]:

$$
\operatorname{RMS}\left(F_{t}\right)=\sqrt{\frac{1}{n} \sum_{i}^{n} F_{x i}^{2}+F_{y i}^{2}} .
$$

The expression for the RMS of the moments shown in (3) and (4) is represented by:

$$
\operatorname{RMS}\left(M_{t}\right)=\sqrt{\frac{1}{n} \sum_{i}^{n} M_{y i}^{2}+M_{z i}^{2}+M_{X i}^{2}} .
$$

First, each of the equations (1)-(7) was individually utilized for optimization process as an objective function $(\mathrm{OF})$, and then a new OF that introduced a combination of the best force and moment $\mathrm{OF}$ was introduced to test all the options that elevated the optimization process. To decide on the best OF, a comparison between balanced and unbalanced (UB) mechanism values is presented using a balancing effect index BI which was introduced by [19]:

$$
\mathrm{BI}=\frac{\text { MAX or RMS value of balanced mechanism }}{\text { MAX or RMS value of unbalanced mechanism }} \text {. }
$$

The idea was to choose the best performance OF based on shaking forces and combine it with the best results of the shaking moment based OF to enhance the optimization process. The root mean square (RMS), maximum (MAX) and balancing effect index (BI), the results of the preliminary optimization process of different OFs on the system's shaking force components (i.e., $F_{x}, F_{y}$ and $F_{t}$ ) are presented in Table 1 . The optimization process was performed using commercial software MSC ADAMS ${ }^{\circledR}$.

Based on these results, it can be noticed that the OF that has the best $\mathrm{BI}$ in terms of shaking forces reduction is the equation representing the total shaking forces $F_{t}$ in (6). Although the BI results for the force component $F_{x}$ and $F_{y}$ are better, the BI value of $F_{t}$ shows the best total reduction, which gives an indication of force component cancellation. At the same time, the shaking moment OF using $M_{z}$ also gives better optimization for the total forces present in the system than that of $M_{t}$, while $M_{x}$ and $M_{y}$ show higher BI values in shaking forces. Similarly, Table 2 presents RMS, MAX, and BI values of different optimization OFs on the system's shaking moments. 
Table 1. RMS, MAX and BI results of shaking force components

\begin{tabular}{l|r|r|r|r|rr}
\hline OF & \multicolumn{2}{|c|}{$F_{x}$} & \multicolumn{2}{c|}{$F_{y}$} & \multicolumn{2}{c}{$F_{t}$} \\
\cline { 2 - 7 } & \multicolumn{1}{|c}{ RMS } & MAX & \multicolumn{1}{c}{ RMS } & MAX & \multicolumn{1}{c}{ RMS } & \multicolumn{1}{c}{ MAX } \\
\hline UB & 2837.65 & 4340.96 & 787.55 & 1316.12 & 2347.34 & 3430.60 \\
$F_{x}$ & 92.29 & 172.96 & 318.54 & 487.94 & 341.84 & 659.92 \\
BI & 0.03 & 0.04 & 0.40 & 0.37 & 0.15 & 0.19 \\
$F_{y}$ & 268.37 & 449.54 & 190.03 & 350.42 & 216.86 & 505.17 \\
BI & 0.09 & 0.10 & 0.24 & 0.27 & 0.09 & 0.15 \\
$F_{t}$ & 258.77 & 350.42 & 265.53 & 444.09 & 238.78 & 459.06 \\
BI & 0.09 & 0.08 & 0.34 & 0.34 & 0.10 & 0.13 \\
$M_{x}$ & 2841.12 & 4013.47 & 1277.72 & 2562.64 & 2380.25 & 430743.00 \\
BI & 1.00 & 0.92 & 1.62 & 1.95 & 1.01 & 125.56 \\
$M_{y}$ & 2998.64 & 4529.70 & 1194.90 & 2318.93 & 2673.80 & 4841.93 \\
BI & 1.06 & 1.04 & 1.52 & 1.76 & 1.14 & 1.41 \\
$M_{z}$ & 195.21 & 350.16 & 238.41 & 386.83 & 253.83 & 536.90 \\
BI & 0.07 & 0.08 & 0.30 & 0.29 & 0.11 & 0.16 \\
$M_{t}$ & 578.13 & 785.24 & 788.15 & 1268.64 & 748.15 & 1079.54 \\
BI & 0.20 & 0.18 & 1.00 & 0.96 & 0.32 & 0.31
\end{tabular}

Using the same concept through the comparison of results, the objective function utilizing $M_{t}$ resulted in the best $\mathrm{BI}$ for reducing shaking moments, indicating that it is the best at the total system's moment reduction. In addition, the objective function of the force component $F_{y}$ also yielded better results than $F_{t}$ OF in balancing the system's shaking moments. Hence, based on the preliminary optimization results shown, it can be decided on the following options as an objective function to enhance the balancing process. These options are:

$$
\begin{gathered}
\mathrm{OF}_{1}: \min \left(F_{t}+M_{t}\right), \\
\mathrm{OF}_{2}: \min \left(F_{t}+M_{z}\right), \\
\mathrm{OF}_{3}: \min \left(F_{y}+M_{t}\right), \\
\mathrm{OF}_{4}: \min \left(F_{y}+M_{z}\right), \\
\mathrm{OF}_{5}: \min \left(F_{t}+F_{y}+M_{t}+M_{z}\right) .
\end{gathered}
$$

Table 2. RMS, MAX, and BI results of shaking moment components

\begin{tabular}{l|l|l|l|l|l|l|lll}
\hline \multirow{2}{*}{ OF } & \multicolumn{3}{|c|}{$M_{x}$} & \multicolumn{3}{c|}{$M_{y}$} & \multicolumn{2}{c|}{$M_{z}$} & \multicolumn{2}{c}{$M_{t}$} \\
\cline { 2 - 9 } & \multicolumn{1}{c}{ RMS } & \multicolumn{1}{c}{ MAX } & \multicolumn{1}{c}{ RMS } & \multicolumn{1}{c}{ MAX } & \multicolumn{1}{c}{ RMS } & \multicolumn{1}{c}{ MAX } & \multicolumn{1}{c}{ RMS } & \multicolumn{1}{c}{ MAX } \\
\hline UB & $1.29 \mathrm{E}+05$ & $3.63 \mathrm{E}+05$ & $1.27 \mathrm{E}+05$ & $2.67 \mathrm{E}+05$ & $2.61 \mathrm{E}+06$ & $3.50 \mathrm{E}+06$ & $2.59 \mathrm{E}+06$ & $3.42 \mathrm{E}+06$ \\
$F_{x}$ & $2.30 \mathrm{E}+05$ & $1.88 \mathrm{E}+05$ & $3.03 \mathrm{E}+05$ & $5.82 \mathrm{E}+05$ & $5.90 \mathrm{E}+06$ & $8.66 \mathrm{E}+06$ & $5.96 \mathrm{E}+06$ & $8.60 \mathrm{E}+06$ \\
BI & 1.78 & 0.52 & 2.39 & 2.18 & 2.26 & 2.47 & 2.30 & 2.51 \\
$F_{y}$ & $2.86 \mathrm{E}+05$ & $4.53 \mathrm{E}+05$ & $3.75 \mathrm{E}+05$ & $7.48 \mathrm{E}+05$ & $1.14 \mathrm{E}+06$ & $1.65 \mathrm{E}+06$ & $1.26 \mathrm{E}+06$ & $1.92 \mathrm{E}+06$ \\
BI & 2.22 & 1.25 & 2.95 & 2.80 & 0.44 & 0.47 & 0.49 & 0.56 \\
$F_{t}$ & $2.27 \mathrm{E}+05$ & $3.55 \mathrm{E}+05$ & $3.06 \mathrm{E}+05$ & $6.19 \mathrm{E}+05$ & $3.15 \mathrm{E}+06$ & $4.61 \mathrm{E}+06$ & $3.14 \mathrm{E}+06$ & $4.51 \mathrm{E}+06$ \\
BI & 1.76 & 0.98 & 2.41 & 2.32 & 1.21 & 1.32 & 1.21 & 1.32 \\
$M_{x}$ & $1.28 \mathrm{E}+05$ & $2.20 \mathrm{E}+05$ & $1.28 \mathrm{E}+05$ & $2.64 \mathrm{E}+05$ & $2.59 \mathrm{E}+06$ & $3.48 \mathrm{E}+06$ & $2.57 \mathrm{E}+06$ & $3.34 \mathrm{E}+06$ \\
BI & 0.99 & 0.61 & 1.01 & 0.99 & 0.99 & 0.99 & 0.99 & 0.98 \\
$M_{y}$ & $1.35 \mathrm{E}+05$ & $2.29 \mathrm{E}+05$ & $1.23 \mathrm{E}+05$ & $2.24 \mathrm{E}+05$ & $5.02 \mathrm{E}+06$ & $7.13 \mathrm{E}+06$ & $5.06 \mathrm{E}+06$ & $7.10 \mathrm{E}+06$ \\
BI & 1.05 & 0.63 & 0.97 & 0.84 & 1.92 & 2.04 & 1.95 & 2.08 \\
$M_{z}$ & $2.50 \mathrm{E}+05$ & $4.03 \mathrm{E}+05$ & $3.36 \mathrm{E}+05$ & $6.57 \mathrm{E}+05$ & $6.83 \mathrm{E}+03$ & $1.32 \mathrm{E}+04$ & $5.00 \mathrm{E}+05$ & $9.22 \mathrm{E}+05$ \\
BI & 1.94 & 1.11 & 2.65 & 2.46 & 0.003 & 0.004 & 0.19 & 0.27 \\
$M_{t}$ & $1.56 \mathrm{E}+05$ & $2.85 \mathrm{E}+05$ & $2.15 \mathrm{E}+05$ & $4.01 \mathrm{E}+05$ & $3.14 \mathrm{E}+04$ & $4.97 \mathrm{E}+04$ & $3.22 \mathrm{E}+05$ & $5.63 \mathrm{E}+05$ \\
BI & 1.21 & 0.79 & 1.69 & 1.50 & 0.01 & 0.01 & 0.12 & 0.16
\end{tabular}


Table 3. DCR mechanism specifications

\begin{tabular}{|c|c|c|c|}
\hline Part & Length (mm) & Mass (kg) & $\begin{array}{l}\text { Moment of inertia IXx, IYY, IzZ, } \\
\qquad\left(\mathrm{kg} \cdot \mathrm{mm}^{2}\right)\end{array}$ \\
\hline $\begin{array}{l}\text { Crank linkages } \\
1,4\end{array}$ & 70.7 & 2.920 & $1.2 \mathrm{E}+04,7071.3,5746.6$ \\
\hline $\begin{array}{l}\text { Coupler } \\
\text { linkages 2, } 5\end{array}$ & 282.8 & 1.958 & $1.6 \mathrm{E}+04,1.6 \mathrm{E}+04,319.5$ \\
\hline $\begin{array}{l}\text { Rocker } \\
\text { linkages } 3,6\end{array}$ & 337.5 & 2.41 & $3.04 \mathrm{E}+04,3.02 \mathrm{E}+04,395.5$ \\
\hline $\mathrm{O}_{1} \mathrm{O}_{2}$ & 470.9 & NA & NA \\
\hline$A_{1} A_{2}$ & 100 & NA & NA \\
\hline
\end{tabular}

Table 4. System design variables

\begin{tabular}{l|c|c|c}
\hline $\begin{array}{c}\text { Design } \\
\text { variables }\end{array}$ & Initial value & Lower limit & Upper limit \\
\hline $\mathrm{CW}_{\mathrm{a}}(\mathrm{kg})$ & 2 & 0.01 & 20 \\
$\mathrm{CW}(\mathrm{kg})$ & 4 & 0.01 & 20 \\
$\mathrm{CW}_{\mathrm{c}}(\mathrm{kg})$ & 3 & 0.01 & 20 \\
$\mathrm{CW}_{\mathrm{d}}(\mathrm{kg})$ & 5 & 0.01 & 20 \\
$\mathrm{D}_{\mathrm{a}, \mathrm{b}}(\mathrm{mm})$ & 0 & 0 & 142 \\
$\mathrm{D}_{\mathrm{c}, \mathrm{d}}(\mathrm{mm})$ & 0 & 0 & 354
\end{tabular}

Referring to Fig. 1, the model of the DCR mechanism has the properties listed in Table 3. The crank angular speed was kept constant at $2000 \mathrm{rpm}$ with no external forces or moments acting on this mechanism.

The design variables considered in the optimization process are introduced in Table 4. Four counterweight masses $\left(\mathrm{CW}_{\mathrm{a}, \mathrm{b}, \mathrm{c}, \mathrm{d}}\right)$ and their corresponding positions $\left(\mathrm{D}_{\mathrm{a}, \mathrm{b}, \mathrm{c}, \mathrm{d}}\right)$ were utilized, the initial values were arbitrary selected.

\section{RESULTS AND DISCUSSION}

In this study, a new approach was applied to study the effect of different optimization parameters on reducing the shaking forces and moments of a DCR mechanism. The objective functions (9)-(13) were used to perform the optimization process where the identified design variables were counterweight masses and positions. There were four counterweights placed along crank and rocker linkages (a, b, c, and d), see Fig. 1. For the five cases using these objective functions in the optimization process introduced in Section 3, the results are shown in Fig. 4.

Shaking forces are presented to compare the results between different objective functions (OF1, OF2, OF3, OF4, and OF5) and the unbalanced (UB) initial status of the DCR mechanism.

The results of the shaking moment components $M_{z}, M_{y}, M_{z}$ and $M_{t}$ from the five cases are compared and plotted in Fig. 5.

The optimization results are presented in Table 5, which indicates the different RMS values of forces and moments resulting from each objective function, as well as the percentage (\%) of reduction in these parameter values.

For better reduction in shaking forces, OF1 and OF3 showed higher reduction percentages where $F_{x}, F_{y}$ and $F_{t}$, were reduced by about $96 \%, 67 \%$ and $89 \%$, respectively. These were followed by OF2, which reduced the total forces $F_{t}$ by about $88 \%$. However, OF4 and OF5 showed the least reduction of these forces. Moreover, in the case of OF4, an increment of the force component $F_{y}$ was recorded by almost $69 \%$, and the incremental values were preceded by the negative sign. 

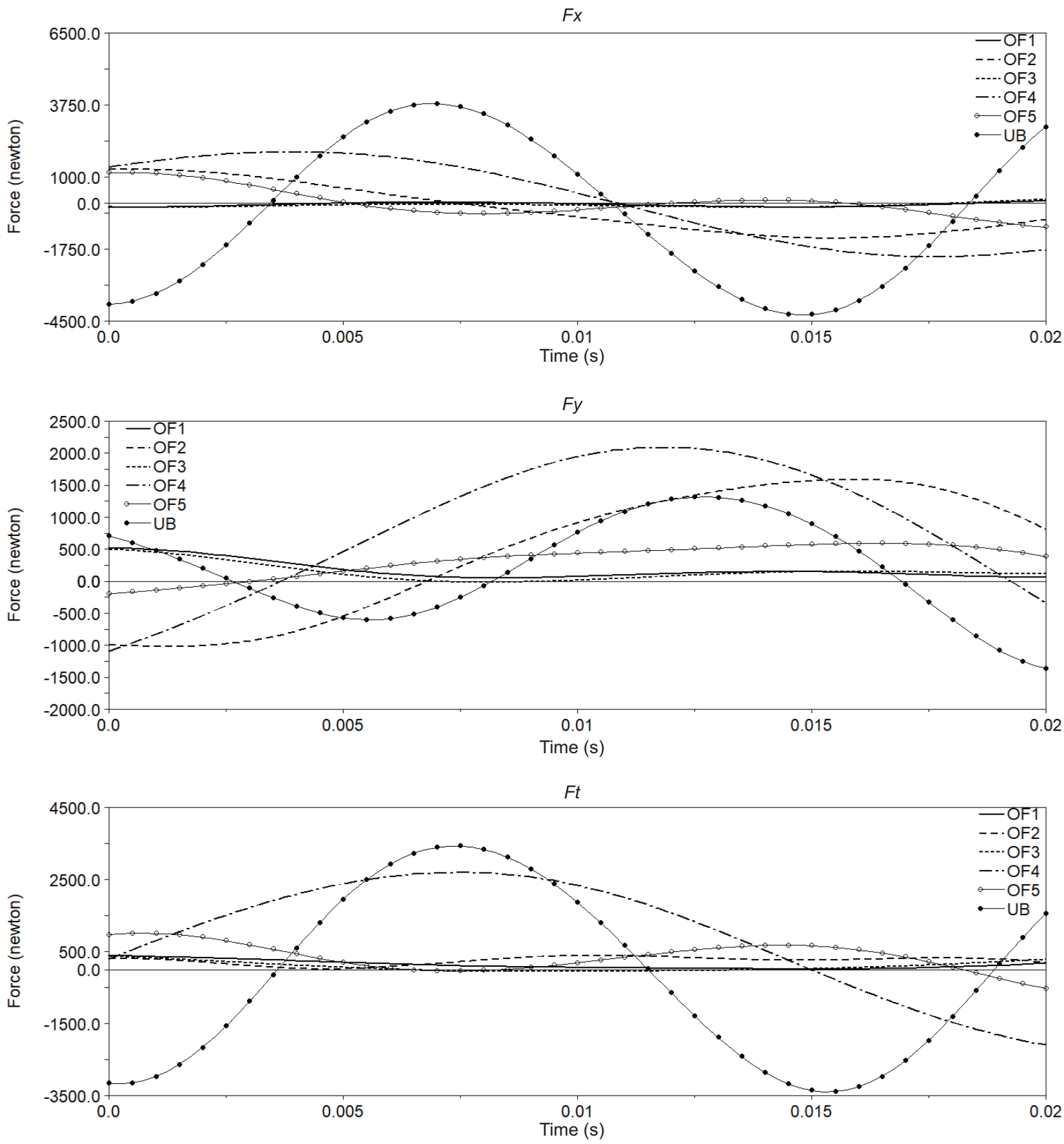

Fig. 4. Unbalanced (UB) and balanced shaking force components for the suggested OF.

Similarly, the total shaking moments caused by this mechanism were highly reduced by OF5 with the reduction of $95 \%$, followed by OF1 and OF3 where almost the same results were obtained; and the reduction in the total shaking moments components $M_{t}$ was about $83 \%$. However, the results of the moment components $M_{x}$ and $M_{y}$ increased in all cases, and the values were preceded by the negative sign. This could be explained by many reasons such as the dominancy of $M_{z}$ as a higher moment acting physically on the system and a larger value presented numerically in the objective function. In addition, the optimization process tends to reduce the shaking effect, and this can be achieved through the counteraction of moment component, even though this might cause increments in the final value of each component. 

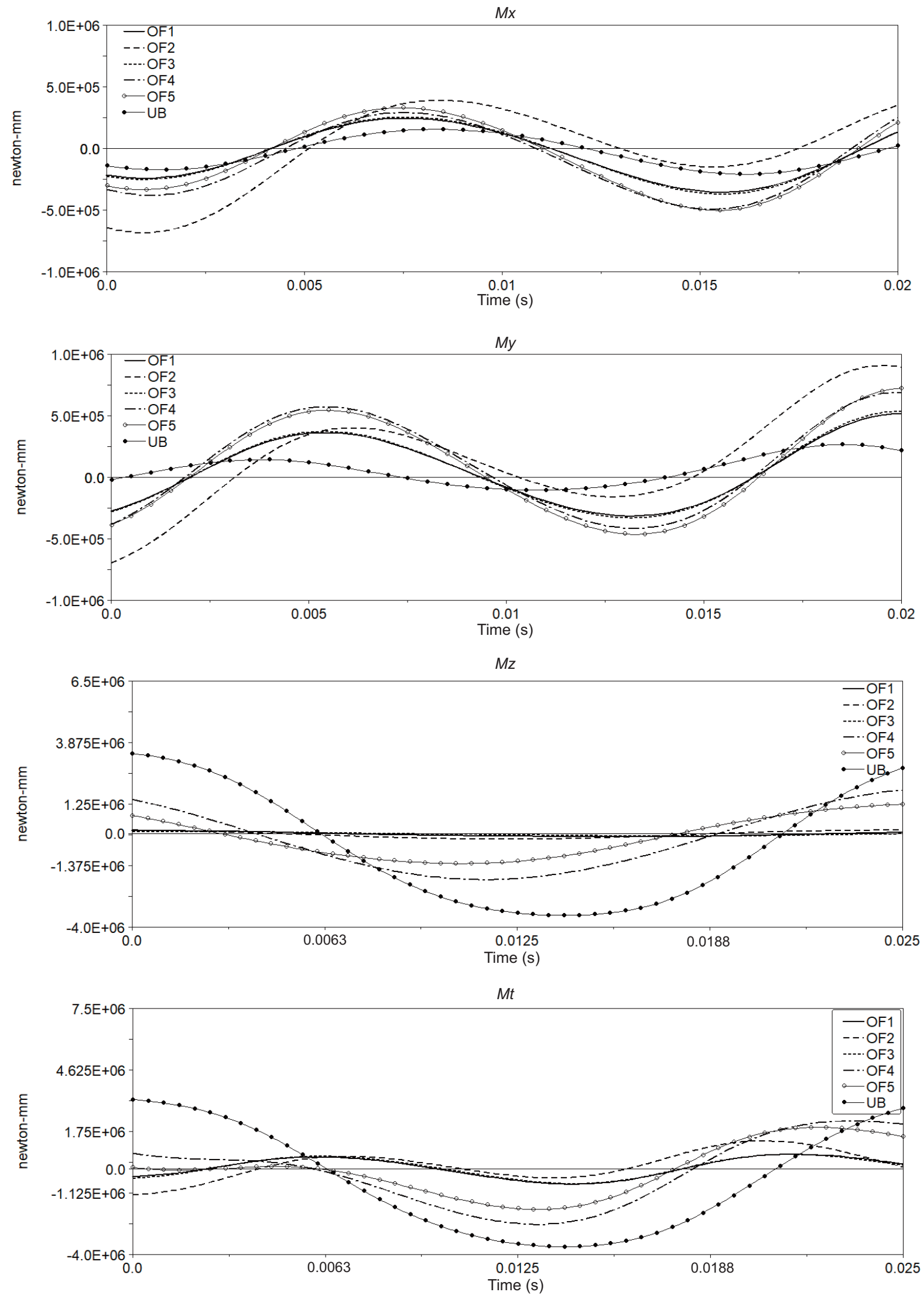

Fig. 5. Unbalanced (UB) and balanced shaking moment components for the suggested OF. 
Table 5. RMS and reduction \% results

\begin{tabular}{llcccccc}
\hline & & OF1 & OF2 & OF3 & OF4 & \multicolumn{1}{c}{ OF5 } \\
\hline$F_{x}$ & RMS & 90.78 & 906.45 & 110.25 & 1467.81 & 578.52 \\
& Reduction\% & 96.801 & 68.056 & 96.115 & 48.274 & 79.613 \\
\hline$F_{y}$ & RMS & 259.34 & 1028.18 & 249.17 & 1337.12 & 387.38 \\
& Reduction\% & 67.070 & -30.554 & 68.361 & -69.782 & 50.812 \\
\hline$F_{t}$ & RMS & 244.2 & 262.72 & 253.95 & 1801.94 & 541 \\
& Reduction\% & 89.597 & 88.808 & 89.181 & 23.235 & 76.953 \\
\hline$M_{x}$ & RMS & $2.10 \mathrm{E}+05$ & $3.37 \mathrm{E}+05$ & $2.17 \mathrm{E}+05$ & $3.03 \mathrm{E}+05$ & $2.95 \mathrm{E}+05$ \\
& Reduction\% & -62.791 & -161.240 & -68.217 & -134.884 & -128.682 \\
\hline$M_{y}$ & RMS & $2.73 \mathrm{E}+05$ & $4.52 \mathrm{E}+05$ & $2.82 \mathrm{E}+05$ & $3.87 \mathrm{E}+05$ & $3.91 \mathrm{E}+05$ \\
& Reduction\% & -114.961 & -255.906 & -122.047 & -204.724 & -207.874 \\
\hline$M_{z}$ & RMS & 92520.16 & $1.44 \mathrm{E}+05$ & 69143 & $1.34 \mathrm{E}+06$ & $8.71 \mathrm{E}+05$ \\
& Reduction\% & 96.455 & 94.483 & 97.351 & 48.659 & 66.628 \\
\hline$M_{t}$ & RMS & $4.24 \mathrm{E}+05$ & $6.72 \mathrm{E}+05$ & $4.31 \mathrm{E}+05$ & $1.51 \mathrm{E}+06$ & $1.11 \mathrm{E}+05$ \\
& Reduction\% & 83.629 & 74.054 & 83.359 & 41.699 & 95.714
\end{tabular}

\section{CONCLUSIONS}

This paper has discussed the impact of different optimization parameters when performing tri-planar balancing enhancement of a DCR mechanism. This system was expected to have better balancing due to its duplicated mechanism, expected to overcome inertial moments caused by its linkages. Therefore, balancing optimization was introduced to minimize other factors causing system vibration, namely reaction forces and moments caused by relevant inertial forces.

A simplified tri-planar dynamic analysis of this mechanism was introduced and presented as a planar mechanism problem. The DCR mechanism model runs with the constant speed of $2000 \mathrm{rpm}$ and has the design variables identified as the masses and positions of the attached counterweights. An approach of choosing the best objective function was presented to solve the optimization problem, and this led to five distinct objective functions (i.e., OF1, OF2, OF3, OF4, and OF5), which were decided based on the balancing effect index BI.

The outcome results show that the reduction percentages related to OF1 and OF3 were the best in eliminating all shaking forces and the moment component $M_{z}$ as these caused an increase in the moment component $M_{y}$ by almost double (the minus sign of reduction percentage represents an increase in the respective value). Likewise, OF2 showed better results in reducing the total shaking forces than OF5, but there was less performance in shaking moment reduction. The overall results of OF4 were less effective in reducing shaking force and shaking moment components than other objective functions.

\section{ACKNOWLEDGEMENT}

The publication costs of this article were partially covered by the Estonian Academy of Sciences.

\section{REFERENCES}

1. Kochev, I. S. General theory of complete shaking moment balancing of planar linkages: A critical review. Mech. Mach. Theory, 2000, 35(11), 1501-1514.

2. Berkof, R. S. Complete force and moment balancing of inline four-bar linkages. Mech. Mach. Theory, 1973, 8(3), $397-410$.

3. Lowen, G. G. and Berkof, R. S. Determination of force-balanced four-bar linkages with optimum shaking moment characteristics. J. Manuf. Sci. Eng. Trans. ASME, 1971, 93(1), 39-46. 
4. Arakelian, V. H. Shaking moment cancellation of self-balanced slider-crank mechanical systems by means of optimum mass redistribution. Mech. Res. Commun., 2006, 33(6), 846-850.

5. Acevedo, M., Orvañanos, T. and Velázquez, R. Shaking moment balancing of a four-bar mechanism using actuation redundancy. Mech. Mach. Sci., 2019, 73, 3319-3327.

6. Arakelian, V. H. Complete shaking force and shaking moment balancing of RSS'R spatial linkages. Proc. Inst. Mech. Eng., Part K: J. Multi-body Dyn., 2007, 221(2), 303-410.

7. Tian, Q., Zhang, Y., Chen, L. and Flores, P. Dynamics of spatial flexible multibody systems with clearance and lubricated spherical joints. Comput. Struct., 2009, 87(13-14), 913-929. http://dx.doi.org/10.1016/j.compstruc.2009.03.006

8. Hai-Yang, Z., Min-Qiang, X., Jin-Dong, W. and Yong-Bo, L. A parameters optimization method for planar joint clearance model and its application for dynamics simulation of reciprocating compressor. J. Sound Vib., 2015, 344, 416-433. http://dx.doi.org/10.1016/j.jsv.2015.01.044

9. Chaudhary, H. and Saha, S. K. An optimization technique for the balancing of spatial mechanisms. Mech. Mach. Theory, 2008, 43(4), 506-522.

10. Albaghdadi, A. M., Baharom, M. B. and Sualiman, S. A. Balancing and simulation of a double crank-rocker engine model for optimum reduction of shaking forces and shaking moments. Math. Model. Eng. Probl., 2021, 8(2), 237-245.

11. Albaghdadi, A. M., Baharom, M. B. and Sulaiman, S. A. Parameter design optimization of the crank-rocker engine using the FMINCON function in MATLAB. IOP Conf. Ser.: Mater. Sci. Eng., 2021, 1088(1), 012072.

12. Stanišić, M. M. Mechanisms and Machines: Kinematics, Dynamics, and Synthesis. 1st edition. Cengage Learning, Stamford, CT, 2015.

13. Sandor, G. N. and Erdman, A. G. Advanced Mechanism Design: Analysis and Synthesis, vol. 2. Prentice-Hall, Englewood Cliffs, NJ, 1984.

14. Yan, H.-S. and Soong, R.-C. Kinematic and dynamic design of four-bar linkages with variable input speed and external applied loads. Trans. Can. Soc. Mech. Eng., 2002, 26(3), 281-310.

15. Erkaya, S. Investigation of balancing problem for a planar mechanism using genetic algorithm. J. Mech. Sci. Technol., 2013, 27(7), 2153-2160.

16. Rao, S. S. Engineering Optimization: Theory and Practice. Fourth edition. John Wiley \& Sons, Hoboken, NJ, 2009.

17. Orvañanos-Guerrero, M. T., Sánchez, C. N., Rivera, M., Acevedo, M. and Velázquez, R. Gradient descent-based optimization method of a four-bar mechanism using fully Cartesian coordinates. Appl. Sci., 2019, 9(19), 4115.

18. Arakelian, V. and Dahan, M. Partial shaking moment balancing of fully force balanced linkages. Mech. Mach. Theory, 2001, 36(11-12), 1241-1252.

19. Lowen, G. G., Tepper, F. R. and Berkof, R. S. The quantitative influence of complete force balancing on the forces and moments of certain families of four-bar linkages. Mech. Mach. Theory, 1974, 9(3-4), 299-323. 